\title{
The Role of SKQ1 (Visomitin) in Inflammation and Wound Healing of the Ocular Surface
}

\author{
Yi Wei (D) Andrew Troger - Viola Spahiu - Natalia Perekhvatova • \\ Maxim Skulachev · Anton Petrov • Boris Chernyak · Penny Asbell
}

Received: October 9, 2018 / Published online: December 18, 2018

(C) The Author(s) 2018

\begin{abstract}
Introduction: SkQ1 (Visomitin) is a novel mitochondrial-targeted antioxidant that holds promise in the treatment of inflammation associated with ocular surface diseases such as dry eye disease (DED) and corneal wounds. However, the specific role of SkQ1 in ocular surface epithelial tissue has yet to be explicated. The goal of this study is to identify roles of SkQ1 in conjunctival inflammation and corneal wound healing.

Methods: To determine the role of SkQ1 in inflammation, human conjunctival epithelial (HCjE) cell cultures were sensitized with proinflammatory cytokine tumor necrosis factor
\end{abstract}

Enhanced digital features To view enhanced digital features for this article go to https://doi.org/10.6084/ m9.figshare.7442534.

Electronic supplementary material The online version of this article (https://doi.org/10.1007/s40123018-0158-2) contains supplementary material, which is available to authorized users.

Y. Wei $(\bowtie) \cdot$ A. Troger · V. Spahiu · P. Asbell

The Icahn School of Medicine at Mount Sinai,

New York, NY, USA

e-mail: ywei21@uthsc.edu

N. Perekhvatova $\cdot$ M. Skulachev $\cdot$ A. Petrov

Mitotech S.A., Luxembourg City, Luxembourg

B. Chernyak

Belozersky Institute of Physico-Chemical Biology,

Moscow State University, Moscow, Russia alpha (TNF- $\alpha$ ) and interleukin 1 beta (IL-1 $\beta$ ) followed by treatments with SkQ1. The production of inflammatory biomarker prostaglandin E2 (PGE2) and cell viability were quantitatively evaluated. To determine the role of SkQ1 in wound healing, human corneal limbus epithelial (HCLE) cell cultures were streaked to create wounds. The wound closure times, ability to support single HCLE cell proliferation and changes of cell migration in the presence of SB203580, an inhibitor of p38 mitogen-activated protein kinase (MAPK), were further compared.

Results: The HCjE and HCLE cultures showed no apparent cytotoxicity to SkQ1 in concentrations up to $250 \mathrm{nM}$ (HCjE) or $2500 \mathrm{nM}$ (HCLE). The HCLE cultures showed no toxicity to SkQ1 at all the SkQ1 concentrations tested. SkQ1 significantly suppressed PGE2 production of $\mathrm{HCjE}$ at concentrations $<300 \mathrm{nM}(24 \mathrm{~h})$ and $50 \mathrm{nM}(48 \mathrm{~h})$, apparently being SkQ1 dose and treatment time dependent. The wound closure rates were increased by $4 \%$ in $4 \mathrm{~h}$ and by $9 \%$ after $8-12 \mathrm{~h}$ in the presence of $50 \mathrm{nM}$ SkQ1. Furthermore, as little as $25 \mathrm{nM}$ of SkQ1 significantly stimulated HCLE single-cell proliferation. Lastly, the SkQ1-stimulated wound healing was completely abolished by SB203580. Conclusion: Results of the current study demonstrate that SkQ1 exhibits an anti-inflammatory role and can be safely applied to ocular surface epithelium up to a concentration of $300 \mathrm{nM}(181 \mathrm{ng} / \mathrm{ml})$ for $24 \mathrm{~h}$. SkQ1 also 
significantly enhances corneal epithelial wound healing, likely through enhancement of cell proliferation and migration. The data provide solid support for SkQ1 as a promising new therapeutic strategy for treatment of conjunctival inflammation as well as corneal wounds.

Funding: This study was sponsored by Mitotech SA Pharmaceuticals.

Keywords: Conjunctiva; Cornea; Epithelial cell cultures; Inflammation; SKQ1; Wound healing

\section{INTRODUCTION}

Reactive oxygen species (ROS), oxidative stress and inflammation have been shown to be important in many eye diseases including dry eye disease (DED), atopic keratoconjuctivitis (AKC), conjunctivochalasis, age-related macular degeneration and glaucoma-related inflammation [1-9]. Oxidative stress is suggested to be a causative factor in the pathogenesis of DED [4] by driving the onset of inflammation and increasing angiogenesis. Recent progress in DED research acknowledges that ocular surface inflammation and damage play etiologic roles and that inflammation is the core mechanism in the pathogenesis of DED [10-14]. Thus, addressing the relationship among ROS production, lipid peroxidation-related membrane damage and the inflammatory process seen in DED may lead to an effective novel approach to DED treatment $[4,14]$.

Oxidative stress has also been confirmed to play important roles in corneal wound healing [15-17]. Wound healing is a recovery process of tissues or organs in response to trauma and injury, beginning with initial inflammatory responses at the wound loci by neutrophils, monocytes and macrophages followed by the formation of granulation tissue with fibroblasts and myofibroblasts and ending with vascularization and epithelization, where ROS has been shown to participate in all three phases $[18,19]$. An unhealed wound is often a result of decreased ROS production and chronic inflammation [20]. A new drug capable of controlling ROS production may hold great promise to achieve perfect wound healing of the cornea.
SkQ1 actively penetrates across the cellular membrane and accumulates in the inner leaflet of the mitochondrial membrane where it is reduced or recharged in a controlled manner. The transition between the two forms helps to reduce damages caused by mitochondrial ROS overproduction [22, 23]. Previous studies on tissue cultures and animal models indicate great therapeutic potentials of SkQ1 and its analogs in diseases with various inflammatory conditions, aging and wound healing [20, 21, 24-31]. Although SkQ1 has been formulated as an eye drop (Visomitin) in Russia and even underwent a small clinical trial for treatment of DED in the USA [32], very little is known about the role and mechanism of SkQ1 in inflammation or wound healing of the ocular surface.

This study explored the roles of SkQ1 in conjunctival epithelial inflammation and corneal epithelial wound healing using in vitro human conjunctival and corneal epithelial cell culture models.

\section{METHODS}

\section{General Methodology}

This article does not contain any studies with human participants or animals performed by any of the authors.

\section{Cells and Culturing}

Human conjunctival epithelial ( $\mathrm{HCjE})$ and corneal epithelial (HCLE) cells were cultured following the protocol provided with the frozen cell stock and consistent with previous publications $[33,34]$. Growth and maintenance were under standard cell culture conditions $\left(37^{\circ} \mathrm{C}, 5 \% \mathrm{CO}_{2}\right.$ and $100 \%$ relative humidity) until confluent, following established protocols [34] (see Supplementary 1 for detailed methodology).

\section{SkQ1 Stock and Working Solutions}

The stock solution of SkQ1, Mitotech-6, batch no. $040414 \mathrm{M}$, was kindly provided by Mitotech S.A. Pharmaceuticals. It was prepared in a solvent containing sterilized 50\% ethanol-0.9\% $\mathrm{NaCl}$ solution. The SkQ1 concentration was 
$100 \mathrm{mg} / \mathrm{ml}$. Following the instructions, this stock was stored at $-20^{\circ} \mathrm{C}$ until use; it can be stored for at least 1 year from the preparation date. The working solutions of SkQ1 were prepared as $2 \times$ serial dilutions in the maintenance medium as described above. Both the stock solution and working dilutions were kept in light-proof containers before and after being applied to the cultures.

\section{Cell Viability, Dosing and Safety Ranges}

\section{Cell Viability Determination with Varying Concentrations of SkQ1 and Diluent}

To evaluate the proper dosing and safety range of SkQ1 on healthy HCjE and HCLE cell cultures, the SkQ1 stock solution from Mitotech SA was serially diluted in culture medium, ranging from $0.25 \mathrm{nM}$ to $250,000 \mathrm{nM}, 10 \times$ range. As a control, the diluent (50\% ethanol) was also serially diluted in culture medium, ranging from 0 to $0.75 \%$, equivalent to the concentrations carrying over from the SkQ1 stock. Cellular morphology changes were observed and recorded by a NIKON D5000 digital camera installed on an Olympus CK2 Inverse Phase Contract Microscope and transferred to a linked computer that ran "Control My Nikon" v3.0 software for further analysis. Quantitative cell viability was determined by the 3-[4,5dimethylthiazol-2-yl]-2,5 diphenyl tetrazolium bromide (MTT) assay protocols established in the laboratory [35]. The MTT assay is based on the conversion of MTT into formazan crystals by living cells, which determines mitochondrial activity optically [36]. The calculated cell viability values were plotted against the SkQ1 concentrations used (see Supplementary 2 for detailed MTT methodology).

\section{Cell Viability Determination when SkQ1 Is

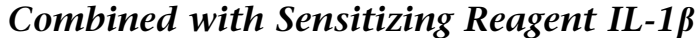 or TNF- $\alpha$}

To rule out potential interferences from the combination of SkQ1 and pre-sensitizing agents on $\mathrm{HCjE}$ cells, the $\mathrm{HCjE}$ cell cultures were presensitized for $1 \mathrm{~h}$ with $10 \mathrm{ng} / \mathrm{ml}$ of TNF- $\alpha$ or IL$1 \beta$ as previously reported [37] and then exposed to various concentrations of SkQ1 in combination with the following: (1) culture medium only; (2) IL-1 $\beta$ (10 ng/ml); (3) TNF- $\alpha$ $(10 \mathrm{ng} / \mathrm{ml})$. Each treatment had three repeats. Controls included medium alone, TNF- $\alpha$ alone, IL-1 $\beta$ alone and SkQ1 alone. Exposure times under the standard incubation were $36 \mathrm{~h}$. Experiments were run on 96-well plates, and results were photographically recorded.

\section{Role of SkQ1 in Inflammation of HCjE Conjunctival Epithelial Cells}

\section{SkQ1 Treatment of HCjE Inflammation}

The HCjE cells were grown in 96-well plates until reaching nearly 95\% confluency, washed twice with pre-warmed medium and then given pre-treatments with freshly made maintenance medium containing varying concentrations of SkQ1 as indicated for $1 \mathrm{~h}$. Then, $1 \mu \mathrm{l}$ of freshly made IL-1 $\beta$ or TNF- $\alpha$ working solution was added to the SkQ1 pre-treated cells at final concentrations of $10 \mathrm{ng} / \mathrm{ml}$. The treatments continued for $24 \mathrm{~h}$. The control wells received the maintenance medium only. To acquire statistically meaningful results, each treatment in one experiment was repeated at least twice, and the entire experiment was repeated at least three times.

\section{PGE2 Assay}

At 24 - or 48 -h SkQ1 treatments, $10 \mu \mathrm{l}$ of samples was taken from each well and immediately analyzed for the concentrations of an inflammatory biomarker, prostaglandin E2 (PGE2), released in the culture medium. A monoclonal enzyme immunoassay (EIA) kit (Cayman Chemical Co., Ann Arbor, MI) was used following the manufacturer's instructions and the protocol established in the laboratory [33]. The degrees of PGE2 production were then calculated and plotted using the formula described in the Methods section.

\section{Role of SkQ1 in Corneal Wound Healing}

\section{Wound Creation}

A single, one-dimensional "streak" defect in the epithelial cell lawn was created in a well by a common scratch method utilizing $200-\mu \mathrm{l}$ or 
1-ml Eppendorf pipette tips, referring to published methods [15, 38, 39].

\section{SkQ1 Treatment Groups}

Immediately after a wound was created, the cultures were gently washed twice with the expired Keratinocyte Serum Free Medium (KSFM, Gibco) to remove remaining dead cell sheets or debris and then re-grown in the maintenance medium. The SkQ1-treated groups received $50 \mathrm{nM}$ SkQ1 in the maintenance medium. The control groups received the medium only with the solvent (ethanol). Since in the preliminary experiments the medium containing ethanol at $0.000002 \%$ concentration, equivalent to the amount that would be carried over from a 50-nM SkQ1 working solution, was tested to be intoxicating to the cells, we omitted the addition of the vehicle solvent to the controls.

\section{End Point Measurements of the Corneal Wound-healing Process}

To monitor and compare the wound-healing processes in the SkQ1-treated groups with those of the control groups, three markers were made alongside each wound at the bottom outside with a fine-tip marker pen to label the loci to be photographed immediately after scratching. These markers were used for alignment purposes: each was photographed together with the individual wounding spot at nearly the same orientation for all the photographing time points at $0,4,8$ and $12 \mathrm{~h}$ from scratching till the wound was completely closed (healing). Photos were taken by a NIKON D5000 digital camera installed on an Olympus CK2 Inverse Phase Contract Microscope. Image files were recorded into a linked computer via "Control My Nikon v3.0" software.

\section{Cell Proliferation}

To evaluate whether SkQ1 has a role in HCLE cell proliferation, extensively diluted single HCLE cells were inoculated into a 24-well plate in the growth and maintenance medium supplemented with various concentrations of SkQ1 ranging from 0 to $400 \mathrm{nM}$. Cell growth was tracked photographically at day $-1,2$ and 6 (corresponding to 24,48 and $144 \mathrm{~h}$ ). A standard MTT assay was conducted, except the stained cells were dried and photographed prior the addition of acidic isopropanol to dissolve formazan and to measure the absorbance at A572 and $690 \mathrm{~nm}$. Two independent experiments were conducted, each with two repeats (see Supplementary 2 for detailed MTT methodology).

\section{Cell Migration}

Cell migration during the corneal epithelial healing process has been demonstrated to be p38 MAPK dependent in an animal model [40]. To evaluate if SkQ1 has a role in HCLE cell migration during the wound-healing process, $10 \mu \mathrm{M}$ of SB203580, an inhibitor of p38 kinase, was added to the maintenance medium together with 0,100 and $400 \mathrm{nM}$ of SkQ1 after scratching. Closure of the wounds was photographically recorded at 0 and $10 \mathrm{~h}$ post-scratch. Two such experiments were conducted independently; each had two repeating wells, and each well had three spots to be photographed.

\section{Data Analysis}

\section{Evaluation of Inflammation on HCjE}

The degree to which PGE2 was produced by HCjE cells sensitized by IL-1 $\beta$ or TNF- $\alpha$ under various concentrations of SkQ1 was compared with the corresponding degree of PGE2 production from cells without sensitization. Data were normalized for interpretation of results. Results were reported as mean $\pm \mathrm{SD}(\sigma)$. A Student's $t$ test was performed for statistical analysis (see Supplementary 3 for specific normalization calculations).

\section{Evaluation of Wound Healing on HCLE}

The areas of wounds at various time points were calculated with ImageJ Fiji software. The whole wounded areas were used for the calculation against a reference with known dimensions, and rates of wound healing (\%) of a specifically marked location at a particular time point were calculated. Results were reported as mean $\pm \mathrm{SD}$ $(\sigma)$ (see Supplementary 3 for specific calculation). 


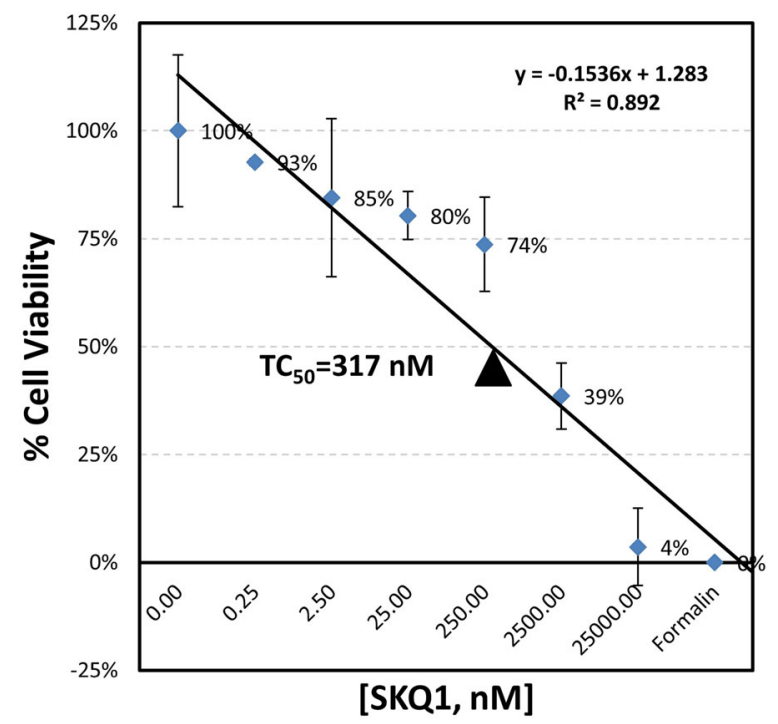

Fig. 1 Calculation of the TC50 value of SkQ1 on $\mathrm{HCjE}$ cells

\section{RESULTS}

\section{Cell Viability, Dosing and Safety Ranges}

\section{Cell Viability Determination with Varying Concentrations of SkQ1 and Diluent}

The cell viability did not significantly decrease until the SkQ1 concentration approached $250 \mathrm{nM}$ (74\% in $\mathrm{HCjE})$ or $2500 \mathrm{nM}$ (85\% in HCLE), as quantitatively measured by MTT analysis. These results were confirmed on $\mathrm{HCjE}$ cells by morphologic observation under the microscope. With either method, the solvent alone (ethanol) did not show any apparent toxicity at any concentrations tested. The toxic concentration for $50 \%$ of cells $\left(\mathrm{TC}_{50}\right)$ where SkQ1 reduces $\mathrm{HCjE}$ cell viability by $50 \%$ was calculated to be $317 \mathrm{nM}$ (Fig. 1); we used $300 \mathrm{nM}$ of SkQ1 for conjunctival inflammation experiments. Since SkQ1 at $2500 \mathrm{nM}$ concentration, much higher than the range (0-400 nM) used for HCLE wound-healing experiments, only reduced HCLE cell viability by $15 \%$, the $\mathrm{TC}_{50}$ for HCLE did not need to be calculated.

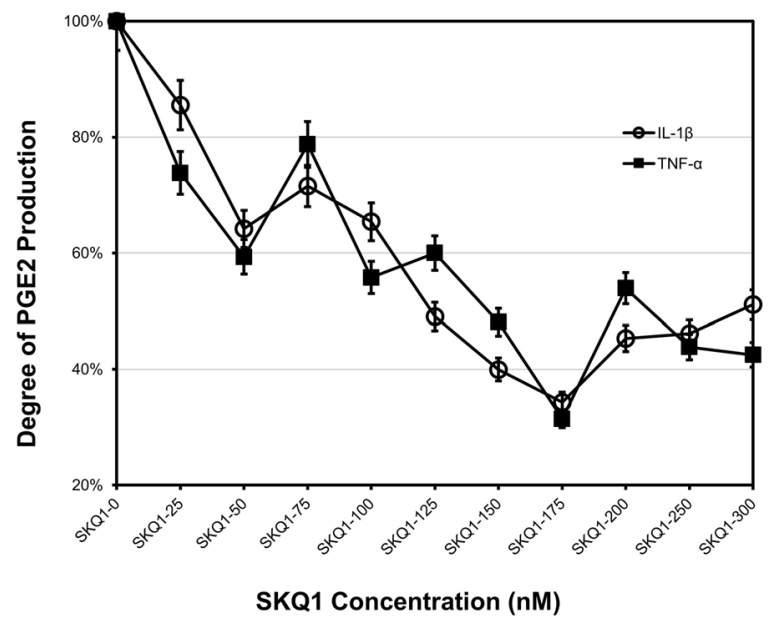

Fig. 2 Role of SkQ1 in conjunctival epithelial inflammation ( $24 \mathrm{~h}$ post-treatment). SkQ1 at concentrations < $300 \mathrm{nM}$ can effectively reduce the PGE2 production in $\mathrm{HCjE}$ as observed at $24 \mathrm{~h}$ of treatment. Data are the mean of four independent experiments, each with duplicate measurements

\section{Cell Viability Determination when SkQ1 Is Combined with Sensitizing Reagent IL-1及 or TNF- $\alpha$}

Regardless of the presence or absence of IL- $1 \beta$ or TNF- $\alpha$, there were no significant color changes in the wells without SkQ1 or in the wells with SkQ1 $\leq 300 \mathrm{nM}$.

\section{Role of SkQ1 in Inflammation of HCjE Conjunctival Epithelial Cells}

Results of four independent experiments are shown in Fig. 2. At the 24-h time point and compared with the untreated controls, the SkQ1-treated cells significantly reduced PGE2 production at concentrations from 25 to $175 \mathrm{nM}$. The strongest inhibitory effects appeared at $175 \mathrm{nM}$, apparently being SkQ1 dose dependent. When the SkQ1 concentrations were further increased, between 200 and $300 \mathrm{nM}$, a strong inhibitory effect was still shown, but the ratios were reduced, perhaps because of the cytotoxicity.

After $48 \mathrm{~h}$ of SkQ1 treatment, the inhibition of PGE2 production was still noticeable, especially when SkQ1 concentrations were $<50 \mathrm{nM}$, apparently being SkQ1 dose dependent (Fig. 3). At concentrations $>50 \mathrm{nM}$, significant 


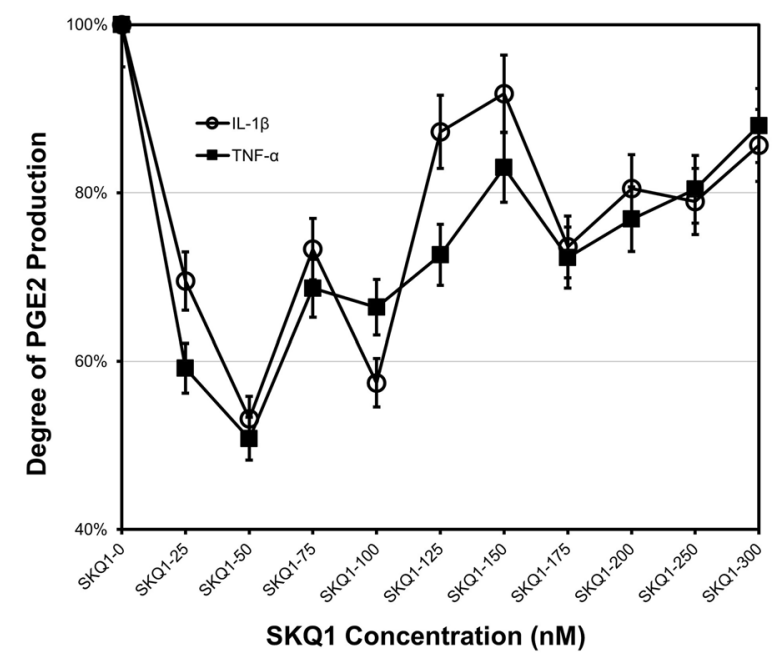

Fig. 3 Role of SkQ1 in conjunctival epithelial inflammation ( $48 \mathrm{~h}$ post-treatment). SkQ1 at concentrations < $50 \mathrm{nM}$ can effectively reduce the PGE2 production in $\mathrm{HCjE}$ as observed at $48 \mathrm{~h}$ of treatment. Data are the mean of four independent experiments, each with duplicate measurements

cytotoxicity was noted, and therefore PGE2 concentrations were not indicative of inhibition by SkQ1.

\section{Role of SkQ1 in HCLE Wound Healing}

SkQ1 in Wound Healing in HCLE Cell Cultures A representative pair of wound-healing results after streaking is shown in Fig. 4, in the presence or absence of $50 \mathrm{nM}$ SkQ1. As shown in Table 1, the addition of $50 \mathrm{nM}$ of SkQ1 significantly stimulated the wound-healing process in HCLE cell cultures: a $4 \%$ increase after $4 \mathrm{~h}$ of SkQ1 treatment $(p=0.015)$ and $9 \%$ increase after $8-12 \mathrm{~h}$ of SkQ1 treatment $(p=0.007$ and 0.003 , respectively).

\section{Role of SkQ1 in HCLE Cell Proliferation}

As shown in Fig. 5a, b, compared with the no SkQ1 controls, the addition of $25-200 \mathrm{nM}$ of SKQ1 significantly stimulated the single cell proliferation in maintenance medium, as indicated by the numbers of the viable cells stained with MTT reagent. Promotion of proliferation was seen clearly yet less significantly when the growth medium was used.

\section{Role of SkQ1 in HCLE Cell Migration}

As shown in Fig. $6 a, b$, in the absence of SB 203580 , the ratios of wounded areas between 0 and $10 \mathrm{~h}$ of treatment were $42 \%$ in the no SkQ1 groups and $25 \%$ in the $400 \mathrm{nM}$ SkQ1-treated groups; the addition of $400 \mathrm{nM}$ SkQ1 contributed a $17 \%$ increase in wound healing. However, when $10 \mu \mathrm{M}$ of SB 203580 was present, the ratios of wound areas between 0 and $10 \mathrm{~h}$ were up to $70 \%$ in the no SkQ1 controls and $69 \%$ in the $400 \mathrm{nM}$ SkQ1-treated groups, nearly completely abolishing the stimulating effect of SkQ1 on HCLE wound closure, with an

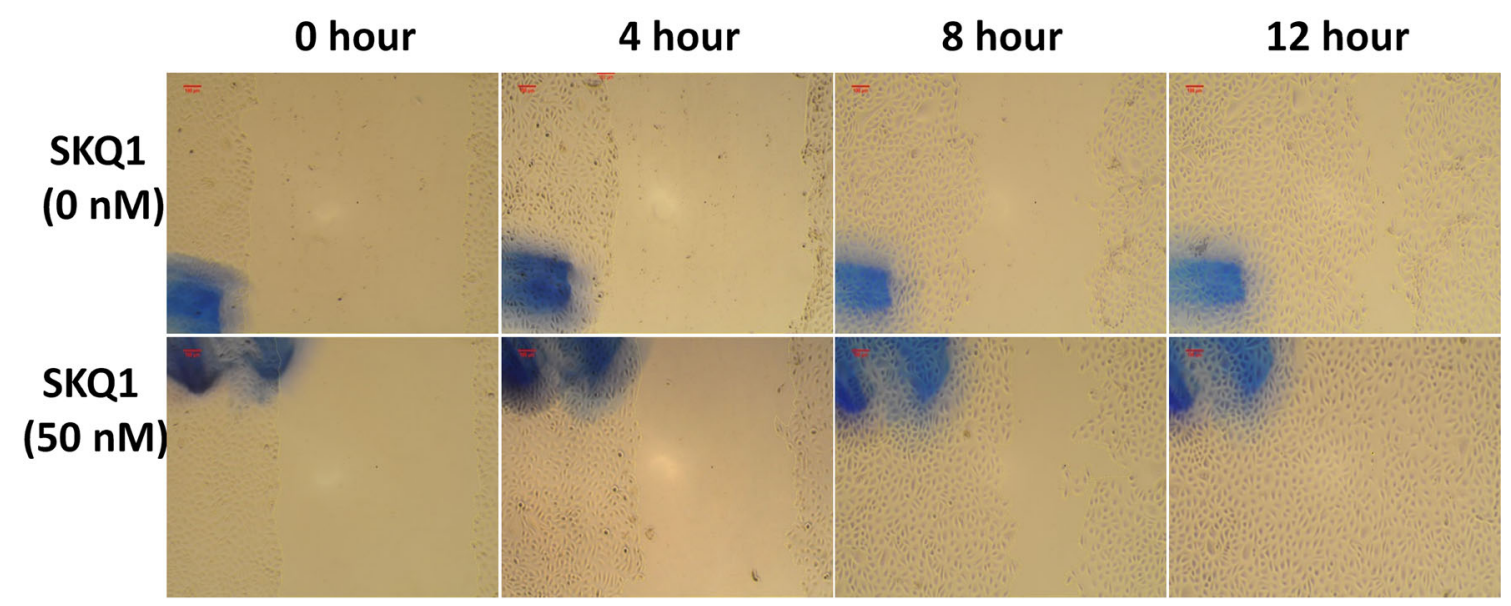

Fig. 4 SkQ1 enhances HCLE wound healing: a representative pair of wound-healing results. The blue markers outside the plate indicate the location where the "wounds" started and are used for line-up over the times 
Table 1 Effect of SKQ1 on wound healing of HCLE cell cultures

\begin{tabular}{|c|c|c|c|c|c|}
\hline \multirow[t]{2}{*}{ Time-points (hours after scratching) } & \multicolumn{2}{|l|}{ SKQ1 at $0 \mathrm{nM}$} & \multicolumn{2}{|c|}{ SKQ1 at $50 \mathrm{nM}$} & \multirow[t]{2}{*}{$p$ value $^{b}$} \\
\hline & $\operatorname{Mean}^{\mathrm{a}} \pm \sigma(\%)$ & $n$ & $\overline{\text { Mean } \pm \sigma(\%)}$ & $\bar{n}$ & \\
\hline 0 & $0 \pm 0$ & 11 & $0 \pm 0$ & 11 & 1 \\
\hline 4 & $17 \pm 6$ & 11 & $21 \pm 5$ & 11 & $0.015^{*}$ \\
\hline 8 & $53 \pm 13$ & 11 & $62 \pm 10$ & 11 & $0.007^{* *}$ \\
\hline 12 & $89 \pm 10$ & 11 & $98 \pm 4$ & 11 & $0.003^{* *}$ \\
\hline
\end{tabular}

a Amount of wound healing $(\%)=\left[1-\frac{\text { (Area of treated wound at a time point) }}{\text { (Area of original wound at time zero) }}\right] x 100$

$\mathrm{b}$ Results of paired two-sample, two-tailed Student's $t$ test for the mean significant differences were established when ${ }^{*} p<0.05$ or ${ }^{* *} p<0.01$ or ${ }^{* * *} p<0.001$

(A)
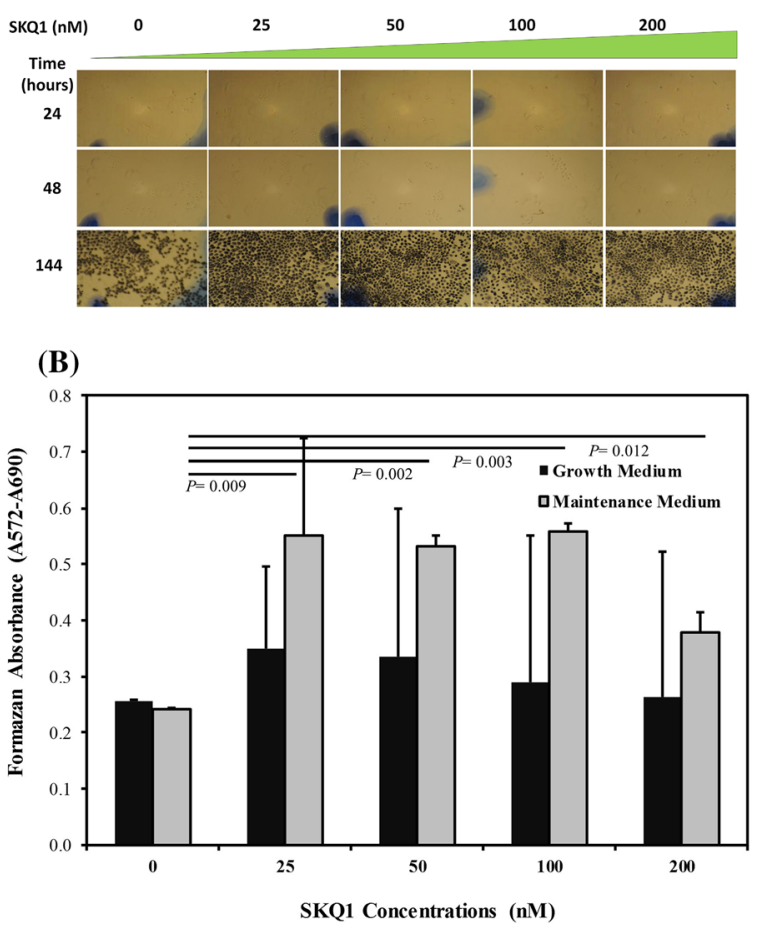

Fig. 5 Role of SkQ1 in HCLE cell proliferation. a The indicated concentrations of SkQ1 were mixed with wellseparated "single" HCLE cells in maintenance medium and inoculated into six-well plates for 6 days until the MTT reagent was added to terminate the experiments. Photos were recorded at 24, 48 and $144 \mathrm{~h}$ post-inoculation. b SkQ1 stimulates HCLE cell proliferation: Results of MTT assays were plotted against the concentrations of SkQ1 used to simulate HCLE cell proliferation

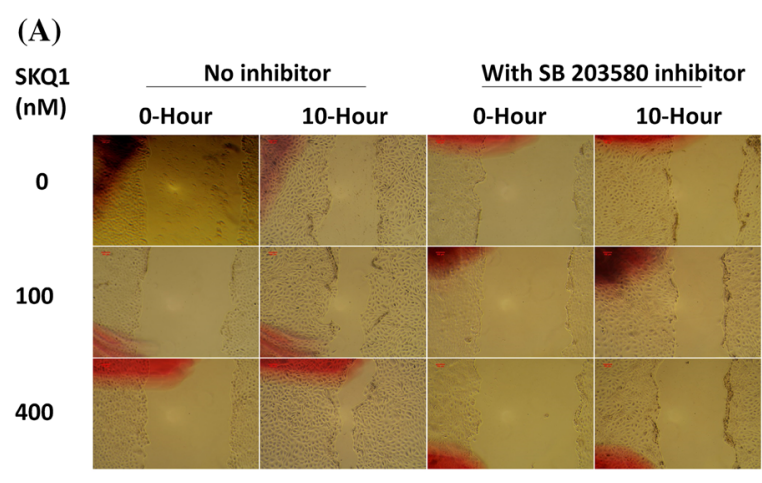

(B)

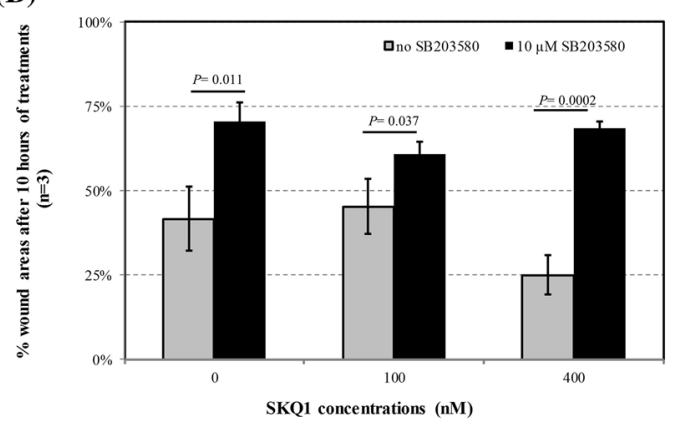

Fig. 6 Role of SkQ1 in HCLE cell migration. a After wounds were created on HCLE cell cultures, SkQ1 at concentrations of 0,100 and $400 \mathrm{nM}$ was added to the maintenance medium in the presence or absence of p38 inhibitor SB203580. The closures of the wounds were photographed at 0 and $10 \mathrm{~h}$ post-SkQ1 treatment. $\mathbf{b}$ The $\%$ wound areas after $10 \mathrm{~h}$ of SkQ1 treatment were plotted against the SkQ1 concentrations 
increase of $29 \%$ in the no SkQ1 groups and $44 \%$ in the $400 \mathrm{nM}$ SkQ1 groups.

\section{DISCUSSION}

In this study, we first demonstrated that SkQ1 can be safely applied to human conjunctival epithelial cells up to a concentration of $300 \mathrm{nM}$ $(181 \mathrm{ng} / \mathrm{ml})$ to reduce PGE2 production (Fig. 1). PGE2 is well recognized as an inflammatory mediator that can be used for quantitative measuring of inflammatory responses of inflamed tissues or cells [37, 41]. Using this established cell culture model, we demonstrated that addition of SkQ1 significantly reduced the production of PGE2 in both TNF- $\alpha$ - and IL- $1 \beta$ sensitized HCjE cells after $24 \mathrm{~h}$ of SKQ1 treatment compared with no-SkQ1 control and in an apparently dose-dependent manner (Fig. 2). The inhibition extended up to $48 \mathrm{~h}$ in concentrations $<50 \mathrm{nM}$, whereas higher concentrations appeared to cause cytotoxicity (Fig. 3). These results provide the first direct evidence that SkQ1 has an anti-inflammatory role in vitro on HCjE cells. We then demonstrated that SkQ1 significantly shortened the wound closure times of the human corneal epithelial cells (Fig. 4 and Table 1) at concentrations of $50 \mathrm{nM}$. Both the epithelial proliferation (Fig. 5) and migration (Fig. 6) were significantly enhanced. These results demonstrated that SkQ1 enhances corneal epithelial wound healing. Taken together, the results of the current study support that SkQ1 is a promising new therapy to inhibit inflammation of the human conjunctival epithelia and promote wound healing of the human corneal epithelia.

The present study provides the first direct evidence that SkQ1 has anti-inflammatory effects in vitro on HCjE cells, and its anti-inflammatory role is likely through participating epithelial innate immunity. SKQ1 showed no apparent toxicity until the concentration approached $250 \mathrm{nM}$. This is consistent with previously published data that demonstrated a SkQ1 eye drop containing $250 \mathrm{nM}$ SkQ1 could effectively prevent and reverse uveitis or even return vision to blind animals [42]. Our studies further demonstrated that increasing concentrations of SkQ1 > $250 \mathrm{nM}$ could cause cellular morphologic changes and cell death. The $\mathrm{TC}_{50}$ was determined to be $317 \mathrm{nM}$. At concentrations $>300 \mathrm{nM}(24 \mathrm{~h})$ or $50 \mathrm{nM}(48$ h), SkQ1 displayed significant cytotoxicity. Therefore, the inhibitory effect on PGE2 production decreased and could not be accurately assessed. This indicates that SkQ1 $<250 \mathrm{nM}$ is biologically safe to human conjunctival epithelial cell cultures. Similarly, SkQ1 appeared not to be cytotoxic to HCLE cells at concentrations $<2500 \mathrm{~nm}$, indicating that SkQ1 at concentrations $<250 \mathrm{~nm}$ is biologically safe for both corneal and conjunctival epithelial cells.

The present study further demonstrated that stimulation of corneal wound healing by SkQ1 was nearly completely abolished in the presence of $10 \mu \mathrm{M}$ SB203580 (Fig. 6). SB203580 is a p38 mitogen-activated protein kinase inhibitor that has been shown to completely suppress wound closure of the corneal epithelial cells [43], indicating the extracellular signal-regulated kinase (ERK) and p38 pathways of the mitogen-activated protein kinase cascade are involved in SkQ1-stimulated HCLE cell migration. It was also interesting to note that the promotion of HCLE cell migration appeared more effective in the presence of $400 \mathrm{nM}$ of SkQ1 and SB 203580 (Fig. 6a) than $400 \mathrm{nM}$ SkQ1 only (no shown), where the cytotoxicity of SkQ1 was clearly seen in the absence of the inhibitor. It is possible that SkQ1 indirectly counter-plays the inhibitory role of SB203580 in p38 kinase via a cellular molecular networking mechanism yet to be determined. It is also possible that SkQ1 accumulates in and has a greater effect on "younger" individual growing cells than "older" and less proliferating cell masses or sheets. SkQ1 appears to be more toxic in proliferating single cells than in the nearly confluent cell sheets. This may imply that the effect of SkQ1 on corneal epithelial wound healing can be affected at least by the cell growth stage as well as the density of the cultures.

Results of the current study are based on human conjunctival and corneal epithelial cell lines. Further studies with primary human conjunctival and corneal cells and appropriate animal models are needed to establish the therapeutic values of SkQ1 in ocular 
inflammatory diseases. Mitochondrial ROS are well known in the regulation of apoptosis under both physiologic and pathologic conditions, especially when cells are inflamed [44]. It will be important to determine the role of SkQ1 in apoptosis of the ocular epithelium.

\section{CONCLUSIONS}

Results presented in this study provide solid evidence to support SkQ1 as a promising new therapy for corneal trauma and wound damages. Given its in vitro efficacy in both conjunctival epithelial inflammation and corneal epithelial wound healing, SkQ1 may have a role as a new anti-inflammatory therapeutic modality in inflammatory conditions of the ocular surface such as chronic allergic conditions or DED. It may also have utility in the context of wound healing, where rapid closure of surface wounds reduces the risk of infection and other vision-threatening complications. Further investigation is warranted to explore the therapeutic potential and underlying mechanistic implications of SkQ1 on ocular surface inflammation and wound healing.

\section{ACKNOWLEDGEMENTS}

The human conjunctival cell line (HCjE) and human corneal epithelial cell (HCLE) line are gifts from Dr. Ilene Gipson at the Schepens Eye Research Institute, Harvard Medical School.

Funding. This study was sponsored by Mitotech SA Pharmaceuticals, 42, rue de la Vallee, 2661 Luxembourg. The article processing charges were funded by the authors.

Editorial Assistance. The authors appreciate the effort of Nancy Lu for manuscript proofreading. Nancy was a volunteer supervised by the corresponding author.

Authorship. All the co-authors meet the international Committee of Medical Journal Editors (ICMJE) criteria for authorship for this article, take responsibility for the integrity of the work as a whole and have given their approval for this version to be published.

Disclosures. Yi Wei, Andrew Troger, Viola Spahiu, Natalia Perekhvatova, Maxim Skulachev, Anton Petrov, Boris Chernyak and Penny Asbell have nothing to disclose.

Compliance with Ethics Guidelines. This article does not contain any studies with human participants or animals performed by any of the authors.

Data Availability. The data sets of the current study are available from the corresponding author on reasonable request.

Open Access. This article is distributed under the terms of the Creative Commons Attribution-NonCommercial 4.0 International License (http://creativecommons.org/licenses/ by-nc/4.0/), which permits any noncommercial use, distribution, and reproduction in any medium, provided you give appropriate credit to the original author(s) and the source, provide a link to the Creative Commons license, and indicate if changes were made.

\section{REFERENCES}

1. Cejkova J, et al. Ocular surface injuries in autoimmune dry eye. The severity of microscopical disturbances goes parallel with the severity of symptoms of dryness. Histol Histopathol. 2009;24(10):1357-65.

2. Seen S, Tong L. Dry eye disease and oxidative stress. Acta Ophthalmol. 2018;96(4):e412-e420. https:// doi.org/10.1111/aos.13526

3. McMonnies C. Reactive oxygen species, oxidative stress, glaucoma and hyperbaric oxygen therapy. J Optom. 2018;11(1):3-9. https://doi.org/10.1016/j. optom.2017.06.002

4. Wakamatsu TH, et al. Evaluation of lipid oxidative stress status in Sjogren syndrome patients. Invest Ophthalmol Vis Sci. 2013;54(1):201-10.

5. Wakamatsu TH, Dogru M, Tsubota K. Tearful relations: oxidative stress, inflammation and eye diseases. Arq Bras Oftalmol. 2008;71(6 Suppl):72-9. 
6. Ward SK, et al. The role of oxidative stress and inflammation in conjunctivochalasis. Invest Ophthalmol Vis Sci. 2010;51(4):1994-2002.

7. Wakamatsu TH, et al. Evaluation of lipid oxidative stress status and inflammation in atopic ocular surface disease. Mol Vis. 2010;16:2465-75.

8. Uchino $\mathrm{Y}$, et al. Oxidative stress induced inflammation initiates functional decline of tear production. PLoS One. 2012;7(10):e45805.

9. Nita M, Grzybowski A. The role of the reactive oxygen species and oxidative stress in the pathomechanism of the age-related ocular diseases and other pathologies of the anterior and posterior eye segments in adults. Oxid Med Cell Longev. 2016;2016:3164734.

10. Stevenson W, Chauhan SK, Dana R. Dry eye disease: an immune-mediated ocular surface disorder. Arch Ophthalmol. 2012;130(1):90-100.

11. Pflugfelder SC, Stern ME, Symposium P. Immunoregulation on the ocular surface: 2 nd cullen symposium. Ocul Surf. 2009;7(2):67-77.

12. Wei Y, Asbell PA. The core mechanism of dry eye disease is inflammation. Eye Contact Lens. 2014;40(4):248-56.

13. Baudouin C, et al. Clinical impact of inflammation in dry eye disease: proceedings of the ODISSEY group meeting. Acta Ophthalmol. 2018;96(2):111119. https://doi.org/10.1111/aos. 13436

14. Craig JP, et al. TFOS DEWS II definition and classification report. Ocul Surf. 2017;15(3):276-83.

15. Wang L, et al. Concentration-dependent effects of transforming growth factor beta1 on corneal wound healing. Mol Vis. 2011;17:2835-46.

16. Liu XF, et al. Nrf2, a potential therapeutic target against oxidative stress in corneal diseases. Oxid Med Cell Longev. 2017;2017:2326178.

17. Deng R, et al. Oxidative stress markers induced by hyperosmolarity in primary human corneal epithelial cells. PLoS One. 2015;10(5):e0126561.

18. Terai $\mathrm{K}$, et al. Crosstalk between TGF-beta and MAPK signaling during corneal wound healing. Invest Ophthalmol Vis Sci. 2011;52(11):8208-15.

19. Chen $\mathrm{L}$, et al. IkappaB kinase beta regulates epithelium migration during corneal wound healing. PLoS One. 2011;6(1):e16132.

20. Demianenko IA, et al. Novel mitochondria-targeted antioxidants, "Skulachev-ion" derivatives, accelerate dermal wound healing in animals. Biochemistry (Mosc). 2010;75(3):274-80.

21. Skulachev VP. Cationic antioxidants as a powerful tool against mitochondrial oxidative stress. Biochem Biophys Res Commun. 2013;441(2):275-9.

22. Zinovkin RA, Bakeeva LE, Chernyak BV, Egorov $\mathrm{MV}$, Isaev NK, Kolosova NG, Korshunova GA, Manskikh VN, Moshkin MP, Plotnikov EY, Rogovin KA, Savchenko AY, Zamyatnin AAJ, Zorov DB, Skulachev MV, Skulachev VP. Mitochondria-targeted antioxidants. Systems biology of free radicals and antioxidants, ed. I. Laher, vol. 2. Berlin, Heidelberg: Springer; 2014.

23. Skulachev VP. Cationic antioxidants as a powerful tool against mitochondrial oxidative stress. Biochem Biophys Res Commun. 2013;441(2):275-9.

24. Nakamura S, et al. Involvement of oxidative stress on corneal epithelial alterations in a blink-suppressed dry eye. Invest Ophthalmol Vis Sci. 2007;48(4):1552-8.

25. Apostolova N, Victor VM. Molecular strategies for targeting antioxidants to mitochondria: therapeutic implications. Antioxid Redox Signal. 2015;22(8):686-729.

26. Dai DF, et al. Mitochondrial oxidative stress in aging and healthspan. Longev Healthspan. 2014;3:6.

27. Demyanenko IA, et al. Mitochondria-targeted antioxidant SkQ1 improves impaired dermal wound healing in old mice. Aging (Albany NY). 2015;7(7):475-85.

28. Chelombitko MA, et al. Comparison of the effects of mitochondria-targeted antioxidant 10-(6'-plastoquinonyl)decyltriphenylphosphonium bromide (SkQ1) and a fragment of its molecule dodecyltriphenylphosphonium on carrageenan-induced acute inflammation in mouse model of subcuteneous air pouch. Bull Exp Biol Med. 2017;162(6):730-3.

29. Demyanenko IA, et al. Mitochondria-targeted antioxidant SkQ1 improves dermal wound healing in genetically diabetic mice. Oxid Med Cell Longev. 2017;2017:6408278. https://doi.org/10.1155/2017/ 6408278

30. Shabalina IG, et al. Improved health-span and lifespan in mtDNA mutator mice treated with the mitochondrially targeted antioxidant SkQ1. Aging. 2017;9(2):315-39.

31. Yang $\mathrm{YH}$, et al. In vivo immunoregulatory properties of the novel mitochondria-targeted antioxidant SkQ1. Mol Immunol. 2012;52(1):19-29. 
32. Petrov A, et al. SkQ1 Ophthalmic solution for dry eye treatment: results of a phase 2 safety and efficacy clinical study in the environment and during challenge in the controlled adverse environment model. Adv Ther. 2016;33(1):96-115.

33. Wei $\mathrm{Y}$, et al. Isoforms of secretory group two phospholipase A (sPLA2) in mouse ocular surface epithelia and lacrimal glands. Invest Ophthalmol Vis Sci. 2012;53(6):2845-55.

34. Hori Y, et al. Effect of retinoic acid on gene expression in human conjunctival epithelium: secretory phospholipase A2 mediates retinoic acid induction of MUC16. Invest Ophthalmol Vis Sci. 2005;46(11):4050-61.

35. Epstein SP, et al. Comparative toxicity of preservatives on immortalized corneal and conjunctival epithelial cells. J Ocul Pharmacol Ther. 2009;25(2):113-9.

36. van Meerloo J, Kaspers GJ, Cloos J. Cell sensitivity assays: the MTT assay. Methods Mol Biol. 2011;731:237-45.

37. Chen D, et al. sPLA2-IIa is an inflammatory mediator when the ocular surface is compromised. Exp Eye Res. 2009;88(5):880-8.

38. Cao L, et al. Downregulation of PTEN at corneal wound sites accelerates wound healing through increased cell migration. Invest Ophthalmol Vis Sci. 2011;52(5):2272-8.

39. Yoon KC, et al. Expression of CXCL9, -10, -11, and CXCR3 in the tear film and ocular surface of patients with dry eye syndrome. Invest Ophthalmol Vis Sci. 2010;51(2):643-50.

40. Saika S, et al. Role of p38 MAP kinase in regulation of cell migration and proliferation in healing corneal epithelium. Invest Ophthalmol Vis Sci. 2004;45(1):100-9.

41. Pruzanski $\mathrm{W}$, et al. Phospholipase A2 in juvenile rheumatoid arthritis: correlation to disease type and activity. J Rheumatol. 1994;21(10):1951-4.

42. Neroev VV, et al. Mitochondria-targeted plastoquinone derivatives as tools to interrupt execution of the aging program. 4. Age-related eye disease. SkQ1 returns vision to blind animals. Biochemistry (Mosc). 2008;73(12):1317-28.

43. Wang Z, et al. Phosphatase-mediated crosstalk control of ERK and p38 MAPK signaling in corneal epithelial cells. Invest Ophthalmol Vis Sci. 2006;47(12):5267-75.

44. Simon HU, Haj-Yehia A, Levi-Schaffer F. Role of reactive oxygen species (ROS) in apoptosis induction. Apoptosis. 2000;5(5):415-8. 